\title{
OBSERVATION OF FIVE-MINUTE-PERIOD GRAVITY WAVES IN THE SOLAR PHOTOSPHERE*
}

\author{
(Invited Review, Abstract)
}

R. T. STEB BIN S

Sacramento Peak Observatory ${ }^{1}$, Sunspot, NM 88349, U.S.A.

PHILIP R. GOODE

Arizona Research Laboratories ${ }^{2,3}$, University of Arizona, Tucson, AZ 85721, U.S.A.

and

HENRY A. HILL

Department of Physics and Arizona Research Laboratories ${ }^{2,3}$, University of Arizona, Tucson, AZ 85721, U.S.A.

\begin{abstract}
Vertically propagating traveling waves have been observed in the solar photosphere. These waves have a period of $278 \pm 41$ seconds and a vertical phase velocity of about $2 \mathrm{~km} \mathrm{~s}^{-1}$. It is noted that these waves also have approximately the same period as the well-studied five-minute-period acoustic mode, which is evanescent in the photosphere. The only consistent interpretation of the traveling waves implies that they are gravity waves. About half the time the gravity waves are outgoing, while the remainder of the time they are ingoing.

The data were collected by Stebbins et al. (1980) to study the vertical structure of the photosphere. They examined velocity pertubations at nine altitudes in the photosphere using a Doppler shift technique. The current work represents a reanalysis of that data which uncovered the five-minute-period traveling waves.

The mean velocity amplitude of disturbances at a given altitude, as registered in the observed Doppler shift, was found to be directly proportional to the mean velocity amplitude at the base of the photosphere. This was the expected relationship between the velocity amplitudes. It was not expected that the standard deviation of the velocity amplitude at a particular altitude would be independent of the velocity amplitude at the

* Proceedings of the 66th IAU Colloquium: Problems in Solar and Stellar Oscillations, held at the Crimean Astrophysical Observatory, U.S.S.R., 1-5 September, 1981.

1 Operated by the Association of Universities for Research on Astronomy, Inc., under contrast AST 78-17292 with the National Science Foundation.

${ }^{2}$ Research supported in part by the U.S. Department of Energy and the National Science Foundation, Astronomy Division.

${ }^{3}$ Arizona Research Laboratories is an interdisciplinary research program at the University of Arizona. The research activities of Hill and Goode are conducted at the Santa Catalina Laboratory for Experimental Relativity by Astrometry (SCLERA), which is administered through Arizona Research Laboratories. SCLERA is jointly operated by Wesleyan University and the University of Arizona.
\end{abstract}


base of the photosphere. In addition, the phase difference between velocities at different altitudes is, unexpectedly, inversely proportional to the velocity amplitude at any altitude. These two traits of the data are consistent with a five-minute-period nonacoustic traveling wave being superimposed on the five-minute-period acoustic mode. This supposition is borne out by a detailed examination of the data in the complex plane of amplitude and phase and by a calculation of the power spectrum of the traveling waves as a function of their vertical wavenumber (see Hill et al., 1982).

\section{References}

Hill, H. A., Goode, P. R., and Stebbins, R. T.: 1982, Astrophys. J. Letters 256, L17.

Stebbins, R. T., Hill, H. A., Zanoni, R., and Davis, R. E.: 1980, in H. A. Hill and W. A. Dziembowski (eds.), Nonradial and Nonlinear Stellar Pulsation, Lecture Notes in Physics, No. 125, Springer-Verlag, Berlin, p. 381 . 